www.jmscr.igmpublication.org

Index Copernicus Value: 79.54

ISSN (e)-2347-176x ISSN (p) 2455-0450

crossrefDOI: https://dx.doi.org/10.18535/jmscr/v7i2.133

\title{
A Case Report of Juvenile Aggressive Ossifying Fibroma [Trabacular Type] in a young Patient
}

\begin{abstract}
Authors
Dr Sajina.S ${ }^{1}$, Dr Binoy Mathews Nedumgottil ${ }^{2}$

${ }^{1}$ Senior Lecturer, Department of Pedodontics \& Preventive Dentistry, Mahe Institute of Dental Sciences and Hospital, Mahe, U.T of Puducherry, India

${ }^{2}$ Assistant Professor, Dept of Prosthodontics, King Faisal University, Al Ahsa - Kingdom Of Saudi Arabia

Abstract

Juvenile aggressive ossifying fibroma is a rare variant of fibro-osseous lesion occurring primarily in children and young adults. It is a benign, but osteogenic neoplasm of bony origin with a potentially aggressive nature. On the account of age of onset, site of occurrence, clinical presentation, histological feature, aggressive nature and increased tendency for recurrence, it is differentiated from the larger group of ossifying fibroma as a separate entity. This article reports a case of juvenile aggressive ossifying fibroma of trabacular pattern in the anterior maxilla of a 6 year old male patient.

Keywords: Trabecular juvenile ossifying fibroma, Fibro-osseous lesions, Maxilla, Aggressive.
\end{abstract}

\section{Introduction}

Juvenile ossifying fibroma (JOF) is described in literature as a rare variant of ossifying fibroma, occurring in the craniofacial skeletal of young individuals $^{[1]}$. Based on the type of mineralized component, two histological variants are recognized namely, Psammomatoid juvenile ossifying fibroma (PsJOF) and Trabecular juvenile ossifying fibroma (TrJOF, WHO (World Health Organisation) Type $)^{[1]}$. The most significant clinical feature that helps to differentiate TrJOF from PsJOF is the site of involvement, with PsJOF occurring mainly in the paranasal sinuses and TrJOF occurring mainly in the maxilla ${ }^{[2]}$. Here one such occurrence of this rare lesion is reported in the maxilla of a 6 year old patient.

\section{Clinical history}

A 6-year-old male child reported to the Department of Pedodontics and Preventive Dentistry, Rajah Muthiah Dental College and Hospital, with th chief complaint of rapidly enlarging mass for past 30 days over the upper front region of jaw. The history of presenting illness revealed that, the swelling started off as a small painless mass that gradually increased in size over a period of one month with mild discomfort along with bleeding while brushing. The past history revealed that, the patient had underwent an excision for a similar lesion at the same site, by a private practitioner elsewhere 6 months back, which recurred. The patient had no contributing family and medical history.

No clinically relevant finding could be detected on extra-oral examination. Intraoral examination 
revealed an asymptomatic ovoid swelling measuring approximately $1.5 \mathrm{~cm} \quad \mathrm{x} \quad 1 \quad \mathrm{~cm}$ extending mesiodistally from mesial half of upper right primary central incisor to the mesial half of upper left primary lateral incisor; superiorly extending upto the marginal gingiva and inferiorly to the level of palatal mucosa with labially displaced upper left primary central incisor. Secondary changes such as redness and mild ulceration were seen over the swelling. There was no evidence of abscess, draining fistulae or sinuses associated with the swelling. On palpation, the swelling was uniformly firm in consistency, non tender and non fluctuant. Hard tissue examination showed Grade I mobility in deciduous right and left central incisors. (51 \& 61)

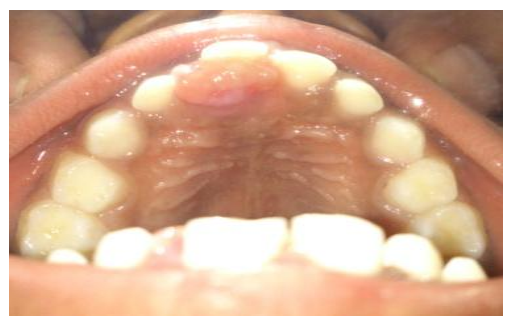

Fig. 1 Preoperative photograph showing the lesion Radiological investigation included an intraoral pe riapical radiograph of the lesion which showed evidence of root resorption of primary teeth (51\&61). Routine hematological evaluation ascertained values within normal limits. Based on the clinical findings and presentation, the differential diagnosis established were fibroma, osteofibrous dysplasia and fibrous dysplasia.

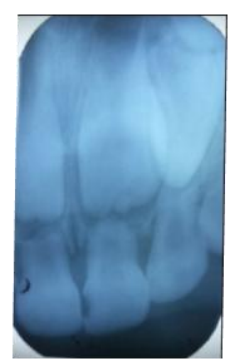

Fig. 2 Intraoral periapical radiograph of 51, 61, 62 showing root resorption of 51, 61

Excisional biopsy was performed under local anes thesia along with the extraction of both the upper central incisors. The excised specimen was fixed in $10 \%$ formalin and sent for histopathological examination. A follow-up examination of the patient after nine months showed no sign of recurrence or relapse. The patient is under regular clinical and radiological follow-ups due to the recurrent nature of the lesion.

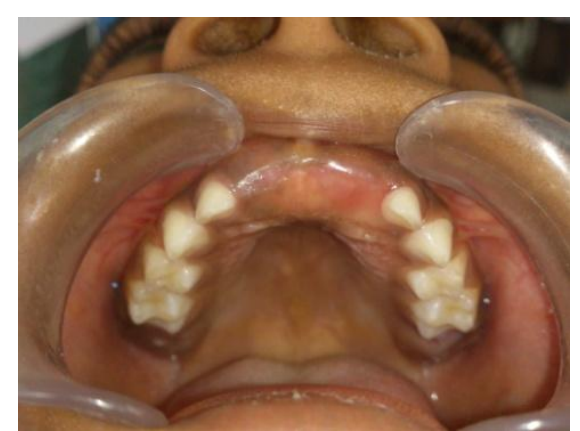

Fig. 4 Postoperative photograph after 6 months Investigation

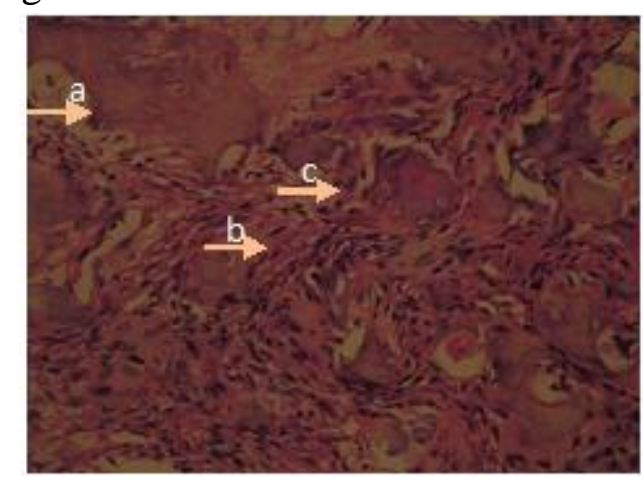

Fig. 5 Photomicrograph showing epithelium and underlying connective tissue with calcifications. (Original magnification $\times 400$ )

Histopathological examination revealed numerous trabeculae of forming bone ['a' in fig.5] in a highly cellular connective tissue. The connective tissue showed monomorphic fibroblast with spindle to plump nuclei ['b' in fig.5] and indistinct cytoplasm. The bony trabeculae showed osteoid matrix in the periphery [' $c$ ' in fig.5]. Few chronic inflammatory cells were also noted all suggestive of juvenile ossifying fibroma of trabecular pattern. All of the above finding pointed towards a definite diagnosis of trabecular variant of juvenile ossifying fibroma.

\section{Discussion}

According to WHO (World Health Organisation) classification (2005), ossifying fibroma most commonly occurs in the 2 nd to 4 th decades with a female predilection. The histological subtypes 
varies in accordance with mean age of the patients. The subtypes includes, JPOF (Juvenile psammomatoid ossifying fibroma) occurring in patients around 20 years, while that of conventional ossifying fibroma occuring in patients around 35 years of age. JTOF (Juvenile trabacular ossifying fibroma) has a still lower mean age range of 8.5-12 years ${ }^{[3]}$.

JTOF also known as trabecular desmoosteoblastoma, affects mainly the jaws of children and adolescents. Only $20 \%$ of the patients are over 15 years of age. In a review of a number of case series the mean age range was found to be 8.5-12 years ${ }^{[4,5,2]}$.

This present case report attempts at the documentation of a rare entity of juvenile ossifying fibroma occurring in a 6 year old male child. The patient reported with a chief complaint of swelling in upper jaw with a previous history of treatment for the same. As the lesion recurred and grew rapidly in the present case, a more aggressive behavior of juvenile ossifying fibroma was provisionally diagnosed which probably occurred due to incomplete initial removal of the lesion from the prior treatment or persistence of some local irritants. The site of occurrence and the histo-pathological picture further confirmed the final diagnosis as a trabecular pattern of juvenile aggressive ossifying fibroma.

The few commonly suggested etiology for the occurrence of aggressive pattern of juvenile ossifying fibroma in young patient are the high levels of periodontal ligament activity (e.g., formation and degradation) and the constant irritation associated with both primary tooth exfoliation and permanent tooth eruption in children $^{[6,7]}$. In the present case, the pre-shedding mobility associated with 51, 61 along with the eruption of 11,21 could be suggested as a possible etiological factor contributing to the recurrence of the lesion after the initial treatment.

Juvenile ossifying fibromas are clinically and histologically distinct from other fibro-osseous lesions, in that it has a recurrence rate of 30 to $56 \%^{[1]}$, thereby necessitating a more radical approach rather than conservative curettage ${ }^{[8,9]}$. Accordingly, in the present case, the line of treatment performed was surgical resection, which was highly effective with no signs of recurrence even after a follow up of 6 months [Figure 4].

The aggressive local behavior of juvenile ossifying fibroma and the rarity of this condition along with the unpredictable recurrence at varied intervals calls for an early diagnosis, prompt treatment, and especially, long-term follow up of the patient. Elimination of etiological factors and complete surgical excision along with involved periodontal ligament and periosteum minimize the possibility of recurrence. The case presented here is an unique variant of juvenile ossifying fibroma of trabacular pattern that has occurred in a very younger age in comparison to the expected age of occurrence and this necessitates the need for further studies for the better understanding of juvenile ossifying fibroma.

\section{References}

1. Bhagwath SS, Sanjaya PR, Babu JS, Koralakunte PK (2014) Trabecular Juvenile Ossifying Fibroma of Mandible. Oral Maxillofac Pathol J 5(2): 514-516.

2. El-Mofty S (2002) Psammomatoid and trabecular juvenile ossifying fibroma of the craniofacial skeleton: Two distinct clinicopathologic entities. Oral Surg Oral Med Oral Pathol Oral Radiol Endod 93: $296-304$.

3. Carvalho B, Pontes M, Garcia H, Linhares P, Vaz R (2012) Ossifying fibromas of the craniofacial skeleton. Histopathology Reviews and Recent Advances 121-132.

4. Slootweg PJ, Panders AK, Koopmans R, Nikkels PG (1994) Juvenile ossifying fibroma: An analysis of 33 cases with emphasis on histopathological aspects. J Oral Pathol Med 23: 385-388.

5. Slootweg PJ, Muller H (1990) Juvenile ossifying fibroma- Report of four cases. J Craniomaxillofac Surg 18(3): 125-129. 
6. Eversole LR, Rovin S (1972) Reactive lesions of the gingiva. J Oral Pathol 1(1): 30-38.

7. Kendrick F, Waggoner WF (1996) Managing a peripheral ossifying fibroma. ASDC J Dent Child 63(2): 135-138.

8. Noffke CE (1998) Juvenile ossifying fibroma of the mandible: An 8 year radiological follow-up. Dentomaxillofac Radiol 27: 363-366.

9. Zama M, Gallo S, Santecchai L, Bertozzi E, De Stefano C (2014) Juvenile active ossifying fibroma with massive involvement of the mandible. Plast Reconstr Surg 113: 970-974. 